\title{
Scalable Upper Bounding Models for Wireless Networks
}

\author{
Jinfeng Du, Muriel Médard \\ Massachusetts Institute of Technology \\ Cambridge, 02139, MA \\ Email: \{jinfeng, medard $\} @$ mit.edu
}

\author{
Ming Xiao and Mikael Skoglund \\ Royal Institute of Technology \\ Stockholm, 10044, Sweden \\ Email: \{mingx, skoglund $\} @$ kth.se
}

\begin{abstract}
The framework of network equivalence theory developed by Koetter et al. introduces a notion of channel emulation to construct noiseless networks as upper/lower bounding models for the original noisy network. This paper presents scalable upper bounding models for wireless networks, by firstly extending the "one-shot" bounding models developed by Calmon et al. and then integrating them with network equivalence tools. A channel decoupling method is proposed to decompose wireless networks into decoupled multiple-access channels (MACs) and broadcast channels (BCs). The main advantages of the proposed method is its simplicity and the fact that it can be extended easily to large networks with a complexity that grows linearly with the number of nodes. It is demonstrated that the resulting upper bounds can approach the capacity in some setups.
\end{abstract}

\section{INTRODUCTION}

A theory of network equivalence has been established in [1], [2] by Koetter et al. to characterize the capacity of a large memoryless noisy network: the original noisy network is first decomposed into many independent single-hop noisy channels; each of the single-hop noisy channels is then replaced by its corresponding upper bounding model (resp. lower bounding model) consisting of only noiseless bit-pipes; the capacity of the resulting noiseless network serves as an upper (resp. lower) bound for the capacity of the original noisy network. A noisy channel $\mathcal{N}$ and a noiseless bit-pipe $\mathcal{C}$ are said to be equivalent if the capacity region of any arbitrary network that contains $\mathcal{N}$ remains unchanged after replacing $\mathcal{N}$ by $\mathcal{C}$. For independent single-hop multi-terminal channels, such as the multiple-access channel (MAC), the broadcast channel (BC), and the interference channel (IC), operational frameworks for constructing upper and lower bounding models have been proposed in [2], and explicit bounding models for 2-user MAC/BC/IC have been constructed in [2]-[4]. The constructive proofs presented in [1], [2] are based on a notion of channel emulation. The lower bounding models (denoted by $\mathcal{C}_{l} \subseteq \mathcal{N}$ ) are established by showing that any code that runs reliably over the stacked network $\mathcal{C}_{l}(N$ parallel replicas of $\mathcal{C}_{l}$ ) can also run over $\underline{\mathcal{N}}$ with similar error probability using channel coding arguments. The upper bounding models $\mathcal{N} \subseteq \mathcal{C}_{u}$ are constructed based on lossy source coding arguments over the stacked networks. A class of bounding models proposed in [5] by Calmon et al. introduces an auxiliary node for each BC/MAC such that the sum rate is characterized by channel emulation over stacked networks as in [1], [2], whilst all individual rates are characterized by emulating the transmission over each channel use (hence named "one-shot").

The bounds obtained by network equivalence tools can be tight in some setups, as shown in [4] for a multiple unicast network composed of 2-user BCs, and in [6] for a frequencydivision AWGN relay network in the wideband regime. However, it is non-trivial to apply the network equivalence tools to bound the capacity of wireless networks owing to the broadcast nature of radio transmission. On one hand, the bounding models proposed in [2] for $m$-user MAC/BC contain $\left(2^{m}-1\right)$ bit-pipes $^{1}$, leading to computational inefficiency when $m$ is large. On the other hand, the received signal at a terminal may contain several broadcasted signals, which creates dependence among several transmitter-receiver pairs. Although such dependence has been partially incorporated into ICs, the whole family of multi-hop channels (e.g., relay channels) have been excluded from consideration since the channel emulation techniques are developed for single-hop channels.

In this paper, we present a simple but efficient method that can construct upper bounding models for wireless networks, at a complexity that grows linearly with the number of nodes. The main advantage of our proposed bounding models are their simplicity and the fact that they can be easily extended to large networks. We demonstrate by examples that the resulting upper bounds can be tight (approaching the capacity) in some setups. Throughout this paper, we assume that the distortion components (e.g., noise) are independent from desired signals. We further assume that the distortion components within a coupled BC are independent, though we still allow noise correlation within a non-coupled $\mathrm{BC}$.

There are other methods aiming at either emulating a noisy channel or characterizing the capacity of wireless networks. For point-to-point channels, the same upper bounding models established in [1] have also been developed in [7] for discrete memoryless channels with finite-alphabet, and in [8] under the notion of strong coordination, where total variation (i.e., an additive gap) is used to measure the difference between the desired and the empirical joint distributions of a pair of sequences. The concept of channel emulation [1], [2], on the other hand, focuses on the set of jointly typical inputoutput pairs and the difference between the empirical joint

\footnotetext{
${ }^{1}$ For an $m$-user IC, the number of bit-pipes in [2] is up to $m\left(2^{m}-1\right)$.
} 
distribution (averaged over ensembles of channel emulators) and the desired distribution is quantified by a multiplicative gap to ensure a small probability of error events. As we focus on characterizing capacity (bounds) rather than reconstructing (exact) common randomness, we shall follow the network equivalence framework [1], [2].

The rest of the paper is organized as follows. We extend the one-shot bounding model to $m$-user MACs/BCs in Sec. II and present the channel decoupling method in Sec. III. We illustrate our upper bounding method in Sec. IV and conclude in Sec. V. Omitted proofs can be found in [9].

\section{Bounding Models FOR NON-COUPLED MACs/BCs}

Given an MAC/BC with $m$ transmitters/receivers, our upper bounding model is represented by a length- $(m+1)$ vector

$$
\mathcal{C}_{u} \triangleq\left(R_{s}, R_{1}, \ldots, R_{i}, \ldots, R_{m}\right)
$$

where $R_{i}$ is the rate constraint on transmitter/receiver $i$, and $R_{s}$ is the sum rate constraint. Constraints on subsets of users,

$$
R(S) \triangleq \sum_{i \in S} R_{i}, S \subset\{1, \ldots, m\}, \text { and }|S| \geq 2,
$$

are omitted, which results in a looser but simpler upper bound. We choose the simple structure for its low complexity and the fact that it facilitates channel decoupling in a natural way.

To simplify notation, for $k=1, \ldots, m$, we represent a collection of $k$ random variables $\left\{X_{1}, X_{2}, \ldots, X_{k}\right\}$ by $X_{[1: k]}$ and their corresponding realizations $\left\{x_{1}, x_{2}, \ldots, x_{k}\right\}$ by $x_{[1: k]}$.

\section{A. MACs with $m$ Transmitters}

For an $m$-user $\operatorname{MAC}\left(\prod_{i=1}^{m} \mathcal{X}_{i}, p\left(y \mid x_{[1: m]}\right), \mathcal{Y}\right)$, two oneshot bounding models have been developed in [5],

$$
\begin{aligned}
& \mathcal{C}_{u, M A C, 1}=\left(R_{s}=R_{M A C}, R_{i}=\log \left(\left|\mathcal{X}_{i}\right|\right), \forall i\right), \\
& \mathcal{C}_{u, M A C, 2}=\left(R_{s}=\log (|\mathcal{Y}|), R_{i}=\max _{p(x)} I\left(X_{i} ; Z_{i}\right), \forall i\right),
\end{aligned}
$$

where $|\mathcal{X}|$ is the cardinality of $\mathcal{X}$ and

$$
R_{M A C}=\max _{p\left(x_{[1: m]}\right)} I\left(X_{[1: m]} ; Y\right) .
$$

Here $Z_{i}$ is the auxiliary random variable for $X_{i}$ such that

$$
p\left(y \mid x_{[1: m]}\right)=\sum_{y=f\left(z_{1}, \ldots, z_{m}\right)} \prod_{i=1}^{m} p\left(z_{i} \mid x_{i}\right),
$$

where $f: \prod \mathcal{Z}_{i} \rightarrow \mathcal{Y}$ is a predefined function to emulate the channel output, and the summation is over all feasible $z_{[1: m]}$.

Note that the two one-shot models focus solely either on the sum rate or on individual rates. We propose here a new upper bounding model $\mathcal{C}_{u, M A C, \text { new }}(\alpha), \alpha \in[0,1]$, as follows

$$
R_{s}=\max _{p\left(v_{[1: m]}\right)} I\left(V_{[1: m]} ; Y\right), R_{i}=\max _{p(x)} I\left(X_{i} ; V_{i}\right)
$$

where $V_{[1: m]}$ are auxiliary random variables with a probability function $p\left(y \mid v_{[1: m]}\right)$ such that

$$
\begin{aligned}
p\left(y, v_{[1: m]} \mid x_{[1: m]}\right) & =p\left(y \mid v_{[1: m]}\right) p\left(v_{[1: m]} \mid x_{[1: m]}\right), \\
p\left(v_{[1: m]} \mid x_{[1: m]}\right) & =\prod_{i=1}^{m} p\left(v_{i} \mid x_{i}\right),
\end{aligned}
$$
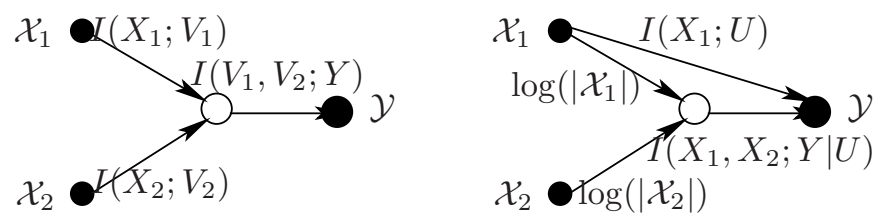

Fig. 1. The general model $\mathcal{C}_{u, M A C \text {, new (left) and the model developed }}$ in [2, Theorem 6] (right) for a 2-user MAC. The label on each bit-pipe is the minimum rate of the corresponding bit-pipe for any given $p\left(x_{1}, x_{2}\right)$.

and $\left\{p\left(v_{i} \mid x_{i}\right), \forall i\right\}$ can be chosen to minimize $\sum_{i} R_{i}$. The parameter $\alpha \in[0,1]$, which indicates how much "noisy effect" has been incorporated into the sum rate constraints $R_{s}$, is determined by $p\left(y \mid v_{[1: m]}\right)$.

Remark 1: $\mathcal{C}_{u, M A C, \text { new }}(\alpha)$ provides a tradeoff between the bounding accuracy on the sum rate and on each of individual rates. It includes the two one-shot models as special cases: setting $\alpha=1$ (all distortion for $R_{s}$ ) will generate $\mathcal{C}_{u, M A C, 1}$, which give us a tighter bound $R_{M A C}$ on the sum rate but looser constraints on all individual rates; setting $\alpha=0$ will produce $\mathcal{C}_{u, M A C, 2}$, which leads to looser bound on the sum rate but tighter bounds on individual rates.

1) 2-user MACs: For 2-user MACs, it is interesting to compare two upper bounding models: $\mathcal{C}_{u, M A C, \text { new }}$ and the model developed in [2, Theorem 6], as illustrated in Fig. 1. On one hand, setting $V_{1}=X_{1}$ and $V_{2}=X_{2}$ in $\mathcal{C}_{u, M A C, \text { new }}$ results in the same upper bounding model as in [2, Theorem 6] when $U=\emptyset$. On the other hand, if we choose $V_{1}=U$ and a deterministic function $f: \mathcal{U} \times \mathcal{V}_{2} \rightarrow \mathcal{Y}$ such that $y=f\left(u, v_{2}\right)$ and $p\left(y, u, v_{2} \mid x_{1}, x_{2}\right)=p\left(u \mid x_{1}\right) p\left(v_{2} \mid x_{2}\right) p\left(y \mid x_{1}, x_{2}\right)$, we have

$$
\begin{aligned}
I\left(X_{1}, X_{2} ; Y \mid U\right) & =H(Y \mid U)-H\left(Y \mid X_{1}, X_{2}, U\right) \\
& =H\left(V_{2} \mid U\right)-H\left(V_{2} \mid X_{2}\right) \\
& \leq H\left(V_{2}\right)-H\left(V_{2} \mid X_{2}\right) \\
& =I\left(X_{2} ; V_{2}\right) \leq \log \left(\left|\mathcal{X}_{2}\right|\right),
\end{aligned}
$$

with equality in (10) if and only if $X_{1}, X_{2}$ (and thus $U, V_{2}$ ) are independent. Combined with the fact that

$$
0 \leq I\left(X_{1}, X_{2} ; Y \mid U\right) \leq \log (|\mathcal{Y}|)=I\left(U, V_{2} ; Y\right),
$$

we can see that $\mathcal{C}_{u, M A C, \text { new }}$ has a tighter bound for user 1 but looser for user 2 .

2) $m$-user Gaussian MAC: Given noise power $\sigma^{2}=1$ at the receiver and transmit power constraint $\gamma_{i}$ for $X_{i}, i=1, \ldots, m$, we construct the new upper bounding model $\mathcal{C}_{u, M A C, \text { new }}(\alpha)$ as follows. Let $Z_{i}, i=0, \ldots, m$, be independent Gaussian random variable with zeros mean and variance $\alpha_{i}>0$ such that $\alpha_{0}=\alpha$ and $\sum_{i=1}^{m} \alpha_{i}=1-\alpha$. By choosing $V_{i}=X_{i}+Z_{i}$ and

$$
Y=V_{1}+\ldots+V_{m}+Z_{0},
$$

we can obtain from (7) that

$$
\begin{aligned}
& R_{s}(\alpha)=\frac{1}{2} \log \left(1+\frac{\left(\sum_{i=1}^{m} \sqrt{\gamma_{i}}\right)^{2}+1-\alpha}{\alpha}\right), \\
& R_{i}(\alpha)=\frac{1}{2} \log \left(1+\frac{\gamma_{i}}{\alpha_{i}}\right), i=1, \ldots, m .
\end{aligned}
$$


One way to determine the noise partitioning parameters $\alpha_{i}$ is to solve the following optimization problem

$$
\begin{array}{cl}
\min _{\alpha_{1}, \ldots, \alpha_{m}} & \sum_{i=1}^{m} \log \left(1+\frac{\gamma_{i}}{\alpha_{i}}\right), \\
\text { subject to } & \sum_{i=1}^{m} \alpha_{i}=1-\alpha, \\
& \alpha_{i}>0 .
\end{array}
$$

It is convex and by Lagrangian methods [10] we have,

$$
\alpha_{i}^{*}=\frac{1}{2}\left(\sqrt{\gamma_{i}\left(\gamma_{i}+4 \mu\right)}-\gamma_{i}\right), i=1, \ldots, m,
$$

where $\mu$ satisfies

$$
\frac{1}{2} \sum_{i=1}^{m}\left(\sqrt{\gamma_{i}\left(\gamma_{i}+4 \mu\right)}-\gamma_{i}\right)=1-\alpha
$$

Although solving this problem in closed-form is challenging, its upper and lower bounds can be determined as shown by Lemma 1 below. Since the LHS of (16) is monotonously increasing with respect to $\mu$, it is simple to find $\mu$ numerically by evaluating (16) within the region specified by Lemma 1 .

Lemma 1: Given $\alpha \in[0,1]$ and all $\gamma_{i}>0$, we have

$\frac{1-\alpha}{m}+\frac{(1-\alpha)^{2}}{m} \frac{1}{\sum_{i} \gamma_{i}} \leq \mu \leq \frac{1-\alpha}{m}+\frac{(1-\alpha)^{2}}{m^{2}} \frac{1}{\min _{i} \gamma_{i}}$,

where both equalities hold if and only if $\gamma_{1}=\ldots=\gamma_{m}$.

From Lemma 2 below, we can see that the freedom of adjusting $\alpha \in[0,1]$ in the optimized noise partition (15) can not improve the sum rate constraint

$$
R_{M A C}=\frac{1}{2} \log \left(1+\left(\sum_{i=1}^{m} \sqrt{\gamma_{i}}\right)^{2}\right) .
$$

This is intuitive as $R_{M A C}$ is achievable when all the source nodes can cooperate.

Lemma 2: Given all $\gamma_{i}>0$, for any $\alpha \in[0,1]$, we have

$$
\min \left\{R_{s}(\alpha), \sum_{i=1}^{m} R_{i}(\alpha)\right\} \geq R_{M A C},
$$

with equality when $\alpha=1$.

\section{B. BCs with $m$ Receivers}

For $m$-user BCs, a straightforward generalization from [5] will give us the following upper bounding models,

$$
\begin{gathered}
\mathcal{C}_{u, B C, 1} \triangleq\left(R_{B C}, \log \left(\left|\mathcal{Y}_{1}\right|\right), \ldots, \log \left(\left|\mathcal{Y}_{m}\right|\right)\right), \\
\mathcal{C}_{u, B C, 2} \triangleq\left(\log (|\mathcal{X}|), R_{1}, \ldots, R_{m}\right), \\
R_{B C}=\max _{p(x)} I\left(X ; Y_{[1: m]}\right), R_{i}=\max _{p(x)} I\left(X ; Y_{i}\right),
\end{gathered}
$$

where $\mathcal{C}_{u, B C, 2}$ is only valid for independent noise at receivers, i.e., when the transition probability can be factorized as

$$
p\left(y_{[1: m]} \mid x\right)=\prod_{i=1}^{m} p\left(y_{i} \mid x\right) .
$$

Below we show step-by-step how to combine the point-topoint channel emulation ${ }^{2}$ developed in [1] with the Covering Lemma [11] and the Joint Typicality Lemma [11] to construct a new and better upper bounding model. Let $\left[l_{1}, l_{2}, \ldots, l_{m}\right]$

\footnotetext{
${ }^{2}$ As previously mentioned, the channel emulation is done over a stacked
} network which consists of $N$ replicas of the original BC. denote a permutation of the $m$ receivers and $\left[Y_{1}, Y_{2}, \ldots, Y_{m}\right]$ be the corresponding channel outputs, we have

$$
\begin{gathered}
\mathcal{C}_{u, B C, \text { new }}=\left(R_{s}, R_{l_{1}}, \ldots, R_{l_{k}}, \ldots, R_{l_{m}}\right), \\
R_{l_{k}}=\max _{p(x)} I\left(X ; Y_{[1: k]}\right), R_{s}=\max _{p(x)} I\left(X ; Y_{[1: m]}\right),
\end{gathered}
$$

where $R_{l_{k}}$ is the rate constraint to receiver $l_{k}$ and $R_{s}$ is the sum rate constraint.

Step I: Fix a channel input distribution $p_{X}(x)$. As defined in [1], let $\hat{A}_{\epsilon}^{(N)}(X)$ be a subset of the "classical" typical set $T_{\epsilon}^{(N)}(X)$ such that for any $x^{N} \in \hat{A}_{\epsilon}^{(N)}(X)$ as the input to the $\mathrm{BC}$, the probability that the corresponding output sequences $\left\{y_{1}^{N}, y_{2}^{N}, \ldots, y_{m}^{N}\right\}$ are not jointly typical with $x^{N}$ is smaller than a predefined threshold ${ }^{3}$. Furthermore, let $p_{Y_{[1: k]}}\left(y_{[1: k]}\right), k=1, \ldots, m$, be marginal distributions obtained from $p\left(x, y_{[1: m]}\right)=p\left(y_{[1: m]} \mid x\right) p_{X}(x)$, and define a series of conditional distributions as follows

$$
p\left(y_{k+1} \mid y_{[1: k]}\right) \triangleq\left\{\begin{array}{cc}
0, & p_{Y_{[1: k]}}\left(y_{[1: k]}\right)=0, \\
\frac{p_{Y_{[1: k+1]}}\left(y_{[1: k+1]}\right)}{p_{Y_{[1: k]}}\left(y_{[1: k]}\right)}, & \text { otherwise. }
\end{array}\right.
$$

Step II: Generate independently at random $2^{N R_{1}^{\prime}}$ sequences $\left\{y_{1}^{N}\left(w_{1}\right): w_{1}=1, \ldots, 2^{N R_{1}^{\prime}}\right\}$, each according to $\prod_{i} p_{Y_{1}}\left(y_{1, i}\right)$. For any sequence $x^{N} \in \hat{A}_{\epsilon}^{(N)}(X)$, by the Covering Lemma,

$\lim _{N \rightarrow \infty} \operatorname{Pr}\left(\exists w_{1} \in\left[1: 2^{N R_{1}^{\prime}}\right]\right.$ s.t. $\left.\left(x^{N}, y_{1}^{N}\left(w_{1}\right)\right) \in T_{\epsilon_{1}}^{(N)}\right)=1$,

if $R_{1}^{\prime}>I\left(X ; Y_{1}\right)+\delta_{1}\left(\epsilon_{1}\right)$ for some $\epsilon_{1}>\epsilon>0$ and $\delta_{1}\left(\epsilon_{1}\right)>0$ that goes to zero as $\epsilon_{1} \rightarrow 0$. Following the channel emulation argument [1], we define a mapping function $\alpha_{1}\left(x^{N}\right)$ as

$\alpha_{1}\left(x^{N}\right)=\left\{\begin{array}{lc}w_{1}, & \text { if } \exists w_{1} \text { s.t. }\left(x^{N}, y_{1}^{N}\left(w_{1}\right)\right) \in T_{\epsilon_{1}}^{(N)}\left(X Y_{1}\right), \\ 1, & \text { otherwise. }\end{array}\right.$

If there is more than one sequence that is jointly typical with $x^{N}$, then $\alpha_{1}\left(x^{N}\right)$ chooses one of them uniformly at random.

Step III: For each sequence $y_{1}^{N}\left(w_{1}\right)$, generate independently $2^{N R_{2}^{\prime}}$ sequences $\left\{y_{2}^{N}\left(w_{1}, w_{2}\right): w_{2}=1, \ldots, 2^{N R_{2}^{\prime}}\right\}$, each according to $\prod_{i} p\left(y_{2, i} \mid y_{1, i}\left(w_{1}\right)\right)$, where $p\left(y_{2} \mid y_{1}\right)$ is defined in (24). Given $w_{1}=\alpha_{1}\left(x^{N}\right)$ which implies

$$
\operatorname{Pr}\left(\left(x^{N}, y_{1}^{N}\left(w_{1}\right)\right) \in T_{\epsilon_{1}}^{(N)}\left(X Y_{1}\right)\right) \rightarrow 1 \text { as } N \rightarrow \infty,
$$

and according to the Joint Typicality Lemma, for all $w_{2} \in[1$ : $2^{N R_{2}^{\prime}}$, we have

$$
\begin{aligned}
& \operatorname{Pr}\left(\left(x^{N}, y_{1}^{N}\left(w_{1}\right), y_{2}^{N}\left(w_{1}, w_{2}\right)\right) \in T_{\epsilon_{2}}^{(N)}\left(X Y_{1} Y_{2}\right)\right) \\
& \quad \geq 2^{-N\left(I\left(X ; Y_{2} \mid Y_{1}\right)+\delta_{2}\left(\epsilon_{2}\right)\right)},
\end{aligned}
$$

for $\epsilon_{2}>\epsilon_{1}$ and some $\delta_{2}\left(\epsilon_{2}\right)>0$ that goes to zeros as $\epsilon_{2} \rightarrow 0$. For $w_{2} \in\left[1: 2^{N R_{2}^{\prime}}\right]$, define an event $E^{T}\left(w_{2}\right)$ as follows

$$
E^{T}\left(w_{2}\right) \triangleq\left(x^{N}, y_{1}^{N}\left(w_{1}\right), y_{2}^{N}\left(w_{1}, w_{2}\right)\right) \in T_{\epsilon_{2}}^{(N)}\left(X Y_{1} Y_{2}\right),
$$

then we have

$$
\operatorname{Pr}\left(\exists w_{2} \text { s.t. } E^{T}\left(w_{2}\right)\right) \geq 2^{N\left(R_{2}^{\prime}-I\left(X ; Y_{2} \mid Y_{1}\right)-\delta_{2}\left(\epsilon_{2}\right)\right)},
$$

which goes to 1 as $N \rightarrow \infty$ if $R_{2}^{\prime}>I\left(X ; Y_{2} \mid Y_{1}\right)+\delta_{2}\left(\epsilon_{2}\right)$.

\footnotetext{
${ }^{3}$ Typical sequences with larger decoding error probability are expurgated.
} 
We also define a mapping function $\alpha_{2}\left(x^{N}, w_{1}\right)$ such that

$$
\alpha_{2}\left(x^{N}, w_{1}\right)=\left\{\begin{array}{lc}
w_{2}, & \text { if } \exists w_{2} \text { s.t. } E^{T}\left(w_{2}\right), \\
1, & \text { otherwise. }
\end{array}\right.
$$

If there is more than one candidate satisfying the joint typicality condition, $\alpha_{2}(\cdot)$ chooses one of them uniformly at random.

Step IV: For $k=3, \ldots, m$, we treat the set of sequences $\left\{y_{1}^{N}\left(w_{1}\right), \ldots, y_{k-1}^{N}\left(w_{k-1}\right)\right\}$ as one unit and repeat Step III, which generates the corresponding sequences $\left\{y_{k}^{N}\left(w_{[1: k-1]}, w_{k}\right): w_{k}=1, \ldots, 2^{N R_{k}^{\prime}}\right\}$, the mapping function $\alpha_{k}\left(x^{N}, w_{[1: k-1]}\right)$, and the rate constraint

$$
R_{k}^{\prime}>I\left(X ; Y_{k} \mid Y_{[1: k-1]}\right)+\delta_{k}\left(\epsilon_{k}\right)
$$

where $\epsilon_{k}>\epsilon_{k-1}$, and $\delta_{k}\left(\epsilon_{k}\right)>0$ that goes to zeros as $\epsilon_{k} \rightarrow 0$.

Step V: Define a channel emulator with codebook

$$
\left\{y_{k}^{N}\left(w_{1}, \ldots, w_{k}\right): k=1, \ldots, m, w_{k}=1, \ldots, 2^{R_{k}^{\prime}}\right\},
$$

an encoder $\alpha\left(x^{N}\right)=\left[\alpha_{1}(\cdot), \ldots, \alpha_{m}(\cdot)\right]$, and a decoder $\alpha_{k}^{-1}\left(w_{[1: k]}\right)$ for receiver $l_{k}, k=1, \ldots, m$. For any input $x^{N}$, $\alpha\left(x^{N}\right)$ generates a sequence $\left(w_{1}, w_{2}, \ldots, w_{m}\right)$ of $N \sum_{i=1}^{m} R_{i}^{\prime}$ bits that are transmitted to the auxiliary node $n_{I}$, which then forwards $\left(w_{1}, \ldots, w_{k}\right)$ (of $N \sum_{i=1}^{k} R_{i}^{\prime}$ bits) to receiver $l_{k}$. At receiver $l_{k}$, the decoding function $\alpha_{k}^{-1}\left(w_{[1: k]}\right)$ selects a sequence from the codebook $\left\{y_{k}^{N}\right\}$ based on the received information bits, i.e.,

$$
\alpha_{k}^{-1}\left(w_{1}, \ldots, w_{k}\right)=y_{k}^{N}\left(w_{1}, \ldots, w_{k}\right) .
$$

Note that the rate constraints in (29) should be satisfied for $k=1, \ldots, m$, and for all $p_{X}(x)$. Let $N \rightarrow \infty$ and $\epsilon_{m} \rightarrow 0^{4}$, the upper bounding model (22) can be characterized by

$$
\begin{aligned}
R_{l_{k}} & =\sum_{i=1}^{k} R_{i}^{\prime}=\max _{p(x)} I\left(X ; Y_{[1: k]}\right), k=1, \ldots, m, \\
R_{s} & =R_{l_{m}}=\max _{p(x)} I\left(X ; Y_{[1: m]}\right) .
\end{aligned}
$$

The second equality in (31) comes from the fact that

$$
I\left(X ; Y_{[1: k]}\right)=I\left(X ; Y_{1}\right)+I\left(X ; Y_{2} \mid Y_{1}\right)+\ldots+I\left(X ; Y_{k} \mid Y_{[1: k-1]}\right) \text {, }
$$

and the first equality in (32) comes from Step V.

Compared to the upper bounding model $\mathcal{C}_{u, B C, 1}$ specified in (19), the new model $\mathcal{C}_{u, B C, n e w}$ maintains the tight sum rate constraint $R_{B C}$ as specified in (21) and meanwhile improves all the individual rate constraints.

Remark 2: There are in total $m$ ! different permutations of $l_{1}, \ldots, l_{m}$, each leading to a different upper bounding model following our construction method. For each of these upper bounding models, the sum rate constraint and one of the individual rate constraints are tight. Depending on the needs, we can select a specific permutation in our design.

Remark 3: For BC with $m=2$ receivers, the proposed upper bounding model has two different layouts $\mathcal{C}_{u, B C, a}=$ $\left(R_{B C}, R_{1}, R_{B C}\right)$ and $\mathcal{C}_{u, B C, b}=\left(R_{B C}, R_{B C}, R_{2}\right)$, where the latter turns out to be equivalent to the model in [2, Theorem 5]. This is not surprising as the channel emulation codebook used

${ }^{4}$ As $0<\epsilon<\epsilon_{1}<\ldots<\epsilon_{m}$, letting $\epsilon_{m} \rightarrow 0$ implies that all of them go to zero. in our construction is generated in the same way as in [2]: superposition encoding. The proof in [2] is restricted for BC with $m=2$ receivers but provides an explicit error analysis. In contrast, our construction is valid for any $m$ but only claims that the error probability can be made arbitrarily small. As a result, a discretization procedure [11] is necessary when extending our results from finite-alphabet channels to more general (e.g., Gaussian) channels ${ }^{5}$.

\section{Channel Decoupling for Coupled Networks}

If a wireless connection between two nodes is part of both a BC and a MAC (i.e., the BC and the MAC are coupled), we can always identify an independent multiple-input multipleoutput sub-network $\left(\prod_{l=1}^{n} \mathcal{X}_{l}, p\left(y_{[1: m]} \mid x_{[1: n]}\right), \prod_{k=1}^{m} \mathcal{Y}_{k}\right)$ that contains the coupled BC/MAC. This sub-network should be the smallest in size in the sense that it does not contain any independent channels. That is, for any non-trivial complementary subsets $\left(S, S^{c}\right)$ and $\left(D, D^{c}\right)$ of $\{1, \ldots, n\}$ and $\{1, \ldots, m\}$, respectively,

$$
p\left(y_{[1: m]} \mid x_{[1: n]}\right) \neq p\left(y_{[D]} \mid x_{[S]}\right) p\left(y_{\left[D^{c}\right]} \mid x_{\left[S^{c}\right]}\right) .
$$

We then partition the original network into decoupled BCs and MACs, whose transaction functions are constructed based on $p\left(y_{[1: m]} \mid x_{[1: n]}\right)$ as follows.

For the decoupled MAC $X_{[S]} \rightarrow Y_{j}$ the marginal distribution $p\left(y_{j} \mid x_{[1: n]}\right)$, more precisely $p\left(y_{j} \mid x_{[S]}\right)$, preserves the possibility of source cooperation (allowing all possible $p(\boldsymbol{x})$ as in the original network). Therefore we choose it to describe the decoupled MAC with received signal $Y_{j}$ and then construct the upper bounding model following the techniques developed in Sec. II-A. Note that we still have a valid upper bound by considering individually each of the decoupled MACs, since

$$
\begin{aligned}
I\left(X_{[1: n]} ; Y_{[1: m]}\right) & =h\left(Y_{[1: m]}\right)-h\left(Y_{[1: m]} \mid X_{[1: n]}\right) \\
& =h\left(Y_{[1: m]}\right)-\sum_{l=1}^{k} h\left(Y_{\left[D_{l}\right]} \mid X_{[1: n]}\right) \\
& \leq \sum_{l=1}^{k} h\left(Y_{\left[D_{l}\right]}\right)-h\left(Y_{\left[D_{l}\right]} \mid X_{[1: n]}\right) \\
& =\sum_{l=1}^{k} I\left(X_{[1: n]} ; Y_{\left[D_{l}\right]}\right),
\end{aligned}
$$

where the inequality is due to the correlation among $Y_{[1: m]}$, and the second equality comes from the assumption that distortion components in coupled BCs are independent, i.e., the exists a set partition $D_{1}, \ldots, D_{k}$ of $\left\{Y_{[1: m]}\right\}$ such that

$$
p\left(y_{[1: m]} \mid x_{[1: n]}\right)=\prod_{l=1}^{k} p\left(y_{\left[D_{l}\right]} \mid x_{[1: n]}\right) .
$$

For decoupled BCs, we introduce a group of axillary random vectors $Z_{i} \triangleq\left[Z_{i, 1}, \ldots, Z_{i, m}\right], i=1, \ldots, n$, and a predefined function $\boldsymbol{y}=g\left(\boldsymbol{z}_{1}, \ldots, \boldsymbol{z}_{n}\right)$, where $\boldsymbol{y} \triangleq\left[y_{1}, \ldots, y_{m}\right]$, such that

$$
p\left(y_{[1: m]} \mid x_{[1: n]}\right)=\sum_{\boldsymbol{y}=g\left(\boldsymbol{z}_{1}, \ldots, \boldsymbol{z}_{n}\right)} \prod_{i=1}^{n} p\left(\boldsymbol{z}_{i} \mid x_{i}\right),
$$

\footnotetext{
${ }^{5}$ As described in [11, Chp. 3.4.1], the transition function of a continuousalphabet channel should be "well-behaved" to facilitate the discretization and quantization procedure. We shall not mention this explicitly in the subsequent sections when applying our results to Gaussian channels.
} 
where $\boldsymbol{z}_{i}$ is the corresponding "output" vector if $X_{i}=x_{i}$ were the only input signal. We then construct upper bounding models for the decoupled $\mathrm{BC}$ based on the transition probability $p\left(\boldsymbol{z}_{i} \mid x_{i}\right)$. Given $\boldsymbol{y}=g\left(\boldsymbol{z}_{1}, \ldots, \boldsymbol{z}_{n}\right)$, and the fact that $X_{i}-X_{j}-Z_{j}$ forms a Markov chain for all $i \neq j$, we have

$$
\begin{aligned}
I\left(X_{[1: n]} ; Y_{[1: m]}\right) & \leq I\left(X_{[1: n]} ; \boldsymbol{Z}_{1}, \ldots, \boldsymbol{Z}_{n}\right) \\
& =h\left(\boldsymbol{Z}_{1}, \ldots, \boldsymbol{Z}_{n}\right)-\sum_{i=1}^{n} h\left(\boldsymbol{Z}_{i} \mid X_{i}\right) \\
& \leq \sum_{i=1}^{n} h\left(\boldsymbol{Z}_{i}\right)-\sum_{i=1}^{n} h\left(\boldsymbol{Z}_{i} \mid X_{i}\right) \\
& =\sum_{i=1}^{n} I\left(X_{i} ; \boldsymbol{Z}_{i}\right),
\end{aligned}
$$

where the equality in (36) comes from (34). Hence focusing on each individual decoupled $\mathrm{BC}$ still gives us a valid upper bound on the original coupled network.

\section{IlLustrative EXAMPLE}

In Fig. 2(a) we focus on a layered multiple-unicast noisy network where $n$ source-destination pairs are assisted by $n$ intermediate relaying nodes. It is a modified version of [4, Fig. 3$]^{6}$ by replacing the orthogonal transmissions to the relaying nodes with MACs to formulate a coupled network. Assuming all transmission channels are identical, the channel in red color is the bottleneck. If all channels are Gaussian with link SNR $\gamma$, we can construct an upper bounding model as shown in Fig. 2(b) by first performing channel decoupling as described in Sec. III, and then substituting the upper bounding models developed in Sec. II to replace $\mathrm{BCs}$ at source nodes by $\mathcal{C}_{u, B C, a}=\left(R_{b}, R, R_{b}\right)$, BCs at intermediate relaying nodes by $\mathcal{C}_{u, B C, b}=\left(R_{b}, R_{b}, R\right)$, and MACs by $\mathcal{C}_{u, M A C, \text { new }}(\alpha)=\left(R_{s}, R^{\prime}, R^{\prime}\right)$, where

$$
\begin{aligned}
R & =\frac{1}{2} \log (1+\gamma), \quad R_{b}=\frac{1}{2} \log (1+3 \gamma), \\
R^{\prime} & =\frac{1}{2} \log (1+\gamma /(1-\alpha) / 2), \\
R_{s} & =\frac{1}{2} \log (1+(4 \gamma+1-\alpha) / \alpha) .
\end{aligned}
$$

Note that although the value of $R_{s}$ and $R_{c}$ in Fig. 2(b) may change by varying $\alpha \in[0,1]$, the red-color link of capacity $R$ is always the bottleneck, and therefore the sum rate of all the parallel unicasts is upper bounded by $R$. This is actually the capacity as each source node can successfully transmit $B$ packets at rate $R$ over $(B n+1)$ transmission blocks (see [9]), which leads to a sum rate

$$
\frac{n B R}{B n+1}=\frac{n R}{n+1 / B} \rightarrow R, \text { when } B \rightarrow \infty .
$$

It worth noting that the cut-set bound of this network is $n R$, which can be arbitrarily larger than $R$ as $n$ goes unbounded.

\section{SUMMARY}

In this work we have extended the upper bounding models for two-user MACs/BCs to many-user scenarios and proposed a channel decoupling method to decompose the coupled network into decoupled BCs/MACs. We have demonstrated that

\footnotetext{
${ }^{6}$ The network of [4, Fig. 3] is obtained from the multiple-unicast network
} in [12, Fig. 1] which consists of point-to-point bit-pipes only.

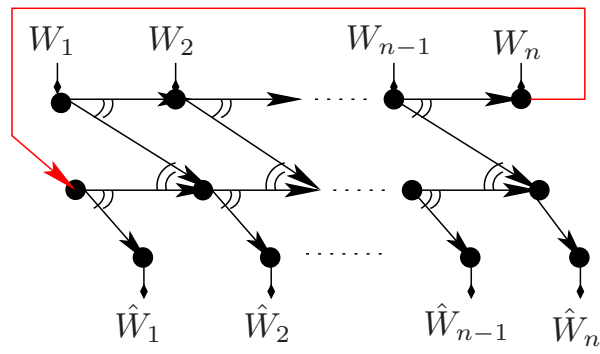

(a) multiple unicast over a coupled noisy network

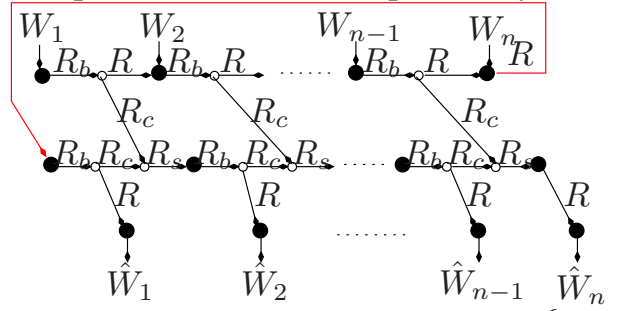

(b) upper bounding model with $R_{c}=\max \left\{R_{b}, R^{\prime}\right\}$.

Fig. 2. Multiple unicast transmission over a coupled noisy network (a) and its upper bounding model (b). All channels are Gaussian with identical link SNR $\gamma$, and the channel in red color is the bottleneck.

the resulting upper bounds can approach capacity in some setups. The proposed methods for constructing upper bounding models, simple and computationally efficient, can be easily extended to large networks. Combined with the lower bounding models [9], they provide additional tools for characterizing the capacity region of general wireless networks.

\section{ACKNOWLEDGMENT}

This work is funded in part by the Swedish Research Council (VR). This material is based upon work supported by the Air Force Office of Scientific Research (AFOSR) under award No. FA9550-13-1-0023.

\section{REFERENCES}

[1] R. Koetter, M. Effros, and M. Médard, "A theory of network equivalence-part I: point-to-point channels," IEEE Trans. Inf. Theory, vol. 57, pp. 972-995, Feb. 2011.

[2] R. Koetter, M. Effros, and M. Médard, "A theory of network equivalence, part II," arXiv:1007.1033, Apr. 2010.

[3] M. Effros, "On capacity outer bounds for a simple family of wireless networks," in Proc. ITA, Feb. 2010.

[4] M. Effros, "Capacity Bounds for Networks of Broadcast Channels," in Proc. IEEE ISIT, Jun. 2010.

[5] F. P. Calmon, M. Médard, and M. Effros, "Equivalent models for multiterminal channels," in Proc. IEEE ITW, Oct. 2011.

[6] N. Fawaz and M. Médard, "A Converse for the Wideband Relay Channel with Physically Degraded Broadcast," in Proc. IEEE ITW, Oct. 2011.

[7] C. Bennett, P. Shor, J. Smolin, and A. Thapliyal, "Entanglement-assisted capacity of a quantum channel and the reverse Shannon theorem," IEEE Trans. Inf. Theory, vol. 48, pp. 2637-2655, Oct. 2002.

[8] P. W. Cuff, H. H. Permuter, and T. M. Cover, "Coordination Capacity," IEEE Trans. Inf. Theory, vol. 56, pp. 4181-4206, Sep. 2010.

[9] J. Du, M. Médard, M. Xiao, and M. Skoglund, "Scalable capacity bounding models for wireless networks," arXiv:1401.4189, Jan. 2014.

[10] S. Boyd and L. Vandenberghe, Convex Optimization, Cambridge University Press, 2004.

[11] A. El Gamal and Y.-H. Kim, Network Information Theory. Cambridge University Press, 2011.

[12] N. Harvey and R. Kleinberg, "Tighter cut-set bounds for $k$-pairs communication problems," in Proc. 43rd Allerton, Sep. 2005. 